\title{
Niveles de plomo en sangre y factores de exposición en niños del estado de Morelos, México
}

Fernando Meneses-González, MD, MSc, ${ }^{(1)}$ Vesta Richardson, MC, (2) Montserrat Lino-González, Lic en Mat,(1) María Teresa Vidal, MD. ${ }^{(2)}$

\section{Meneses-González F, Richardson V, Lino-González M,Vidal MT. Niveles de plomo en sangre y factores de exposición en niños del estado de Morelos, México. Salud Publica Mex 2003;45 supl 2:S203-S208. Este artículo también está disponible en: http://www.insp.mx/salud/index.html}

\section{Resumen}

Objetivo. Evaluar los niveles de plomo en sangre de niños morelenses y sus factores de exposición. Material y métodos. Estudio transversal para analizar, por voltametría anódica, los niveles de plo mo en sangre de 232 niños de 1 a 12 años de edad, que acudieron de junio a octubre de 1996 al Hospital del $\mathrm{N}$ iño Morelense de Cuernavaca, Morelos, México. Los factores de exposición se indagaron por cuestionario. El valor de concentración de plomo se transformó al logaritmo natural; se estimó la razón de momios para algunos factores de exposición que se incorporaron a un modelo de AN OVA. Resultados. Se reclutaron 232 niños ( $50 \%$ mujeres); $73 \%$ residentes en Cuernavaca. La media geométrica de plomo en sangre fue $6.7 \mu \mathrm{g} / \mathrm{dl} ; 29.7 \%$ rebasaron los $10 \mu \mathrm{g} / \mathrm{dl}$; $66 \%$ tenían antecedente de cocinar alimentos en barro vidriado; $36 \%$ de almacenar alimentos, y $19 \%$, consumo de líquidos en ese material. Conclusiones. Los niveles encontrados son similares a los reportados en otras po blaciones pediátricas mexicanas en los últimos años. Entre los principales factores de exposición destacan el uso de barro vidriado para consumo de alimentos o líquidos y la intensidad del tráfico donde viven. Este es el primer estudio que documenta los niveles de plomo en sangre en población infantil de Morelos, México, y sus resultados son

\author{
Meneses-González F, Richardson V, \\ Lino-González M,Vidal MT. \\ Blood lead levels and exposure factors \\ in children of Morelos State, Mexico \\ Salud Publica Mex 2003;45 suppl 2:S203-S208. \\ This paper is available too at: \\ http://www.insp.mx/salud/index.html
}

\begin{abstract}
A bstract
Objective. To assess blood lead levels and lead exposure factors in children living in Morelos State, Mexico. Material and Methods. A cross-sectional study was conducted between June and 0 ctober 1996, in 232 children aged 1-12 years, at Hospital del $\mathrm{N}$ iño Morelense de Cuernavaca, Morelos, Mexico. Blood lead levels were measured by anodic voltameter, and exposure factors were collected by questionnaire. The lead concentration value was log transformed for statistical analysis. 0 dds ratios were obtained for some risk factors.T he statistical significative risk factors were later analyzed with AN OVA. Results. A total of 232 children were recruited (50\% female); $73 \%$ resided in Cuernavaca City.The geo metric mean blood lead level was $6.7 \mu \mathrm{g} /$ $\mathrm{dl} ; 29.7 \%$ of the children had levels over $10 \mu \mathrm{g} / \mathrm{dl} ; 66 \%$ reported use of lead glazed pottery for cooking, $36 \%$ for storing food, and $19 \%$ for drinking. Conclusions. Blood lead levels were similar to those reported in other Mexican children studies, after the reduction of lead in gasoline. The main risk factors were use of lead glazed pottery and vehicle traffic intensity near the household. These results will be useful for future prevention and control interventions. This paper is available too at: http://www.insp.mx/salud/ index.html
\end{abstract}

Este proyecto fue financiado por el Instituto N acional de Salud Pública y el Hospital del N iño Morelense del Gobierno del estado de Morelos.

(1) Centro de Investigación en Salud Poblacional. Instituto N acional de Salud Pública. Cuernavaca, Morelos, México.

(2) Hospital del N iño Morelense. Cuernavaca, Morelos, México.

Fecha de recibido: 12 de septiembre de 2001 • Fecha de aprobado: 7 de junio de 2002

Solicitud de sobretiros: Dr. Fernando Meneses-González. Subdirección de A poyo A cadémico. Centro de Investigación en Salud Poblacional. Instituto N acional de Salud Pública. Avenida U niversidad 655, colonia Santa María A huacatitlan, 62508 C uernavaca, Morelos, México.

Correo electrónico: fmeneses@ correo.insp.mx 
punto de partida para acciones futuras de control y prevención. Este artículo también está disponible en: http:// www.insp.mx/salud/index.html

Palabras clave: niño; plomo; sangre; contaminantes ambientales; México
Key words: child; lead; blood; environmental pollutants; Mexico
D urante los últimos años en México se han reducido de manera importante las fuentes de exposición no ocupacional a plomo, entre las que destacan la de la gasolina, así como la de las latas de conserva de alimentos, pinturas y juguetes, entre otros. Uno de los factores de exposición no ocupacional a plomo que persiste es la loza de barro vidriada con greta de plomo. ${ }^{1}$ A pesar de la formulación de normas para el control de este material en la elaboración de loza vidriada, ${ }^{2}$ aparentemente continúa siendo popular en los talleres de fabricación de este tipo de vasijas y, el uso, aún de las técnicas de "cura" de la vasija de barro en casa, hace que los niveles de plomo persistan de manera importante. ${ }^{3}$ Los efectos para la salud que provoca la exposición a este metal, tanto aguda como crónica, obligan a una especial atención en la población infantil, con el fin de controlar, tanto exposición, como efecto. ${ }^{4}$ Diversos estudios en México han documentado los niveles de plomo en sangre (NPS), tanto en población infantil, como en la adulta, pero no así en el estado de Morelos y en la ciudad de Cuernavaca. Esta ciudad ha tenido un crecimiento urbano acelerado que se refleja en el número de vehículos registrados; del total, $62 \%$ son de uso particular, ${ }^{5}$ sin un programa de control vehicular de emisiones de contaminantes. Asimismo, es una entidad donde se fabrica y distribuye loza de barro vidriada a baja temperatura, ${ }^{*}$ que se estima es de amplio uso en las familias, debido al bajo nivel de precio en el mercado. Puesto que no se conoce la exposición a plomo de la población infantil de esta entidad el presente trabajo se dirigió a establecer los NPS en una muestra de niños que asisten al Hospital del Niño Morelense, así como algunos de los factores de exposición que expliquen las cifras encontradas.

\section{Material y métodos}

Se realizó un estudio transversal, de junio a octubre de 1996, en una muestra de 232 niños de 1 a 12 años de

\footnotetext{
* Tlayacapan, en algún lugar de Morelos. http://www.morelos directo.com/.
}

edad que acudieron a la consulta externa del Hospital del Niño Morelense en Cuernavaca, Morelos, México. La selección de los niños fue aleatoria, sobre la base de un muestreo sistemático: uno de cada cinco niños que acudieron a la consulta del niño sano fueron invitados a participar mediante la autorización de los padres. Se aplicó un cuestionario a las madres para determinar los principales factores de exposición al metal por parte del niño y, además, se obtuvo una muestra de sangre venosa por punción cubital, para determinar los niveles de plomo, con técnica y equipo libre de plomo. La muestra fue procesada en el laboratorio del hospital utilizando un equipo de Voltametría Anódica (Analizador de Metales Traza. Mod.3010A. ESA). El control de calidad y entrenamiento técnico para la toma de las muestras los realizó el Laboratorio de Plomo del ABC. La correlación de un grupo de muestras del estudio con el laboratorio de referencia fue adecuada (90\%). Se realizó un análisis univariado, expresando en proporciones las variables de interés. Para el análisis bivariado se consideró como punto de corte $10 \mu \mathrm{g} / \mathrm{dl}$, siguiendo la recomendación de la Academia Americana de Pediatría, ${ }^{6}$ y se transformó el valor de plomo en sangre al logaritmo natural. Se calcularon las probabilidades para los principales factores de riesgo con base en razones de momios $(R M)$ y se calcularon límites de confianza de 95\% (LC 95\%). Asimismo, las variables significativas resultantes del análisis bivariado fueron incorporadas a un modelo de predicción tipo ANOVA. Se utilizó como programa de análisis Stata v 5.0.*

\section{Resultados}

Fueron reclutados 232 niños cuya media de edad fue de 6.6 años ( $\mathrm{DE}=3.0$ años; rango=1-12 años); $60.6 \%$ fueron mayores de cinco años. El 50\% de la población estudiada fueron mujeres. De todos ellos, $73.3 \%$ procedían del municipio de Cuernavaca y el resto de otras partes del estado. En 76\% de las casas donde se ubica

\footnotetext{
* Statacorp 1996. Stata software: release 5.0 Colege station. TXC.
} Stata Corp.USA. 
el domicilio de los niños el tráfico vehicular era de intensidad media o baja y en el resto era alta. Sólo 17\% de los niños reportaron vivir cerca de un taller de herrería y $10.6 \%$ de un taller mecánico. Con relación al uso de barro vidriado, en $66.8 \%$ las madres lo utilizan para cocinar; $36.4 \%$ para guardar el alimento y consumirlo después, y sólo $8.2 \%$ lo utilizan para tomar líquidos.

La media de plomo en sangre fue de $8.20 \mu \mathrm{g} / \mathrm{dl}$ $(\mathrm{DE}=5.6 \mu \mathrm{g} / \mathrm{dl}$ ) y la media geométrica $6.68 \mu \mathrm{g} / \mathrm{dl} ; 29.7 \%$ de los niños reportaron un NPS por sobre los $10 \mu \mathrm{g} / \mathrm{dl}$. Los niños de 2, 3, 5 y 6 años de edad reportaron los niveles medios de plomo en sangre más elevados (cuadro I). No se encontraron diferencias de acuerdo con el sexo.

Aquellos niños cuyo principal medio de transporte fue el colectivo se les determinó una media del logaritmo de plomo en sangre $(\log \mathrm{PbS})$ de $1.9 \mu \mathrm{g} / \mathrm{dl}$, la cual no tuvo una diferencia estadística cuando se compara con uso de otro tipo de vehículo para transporte. Por otro lado, aquellos que viven en áreas donde la intensidad del tráfico es alta se les determinó una media de $\log \mathrm{PbS}$ de $2.09 \mu \mathrm{g} / \mathrm{dl}$, que fue mayor en comparación con aquellos que viven en zona con intensidad de tráfico baja $(t=-2.67 ; p=<0.05)$. El que la casa del niño se encontrase en avenidas o carreteras no reportó diferencias en los NPS.

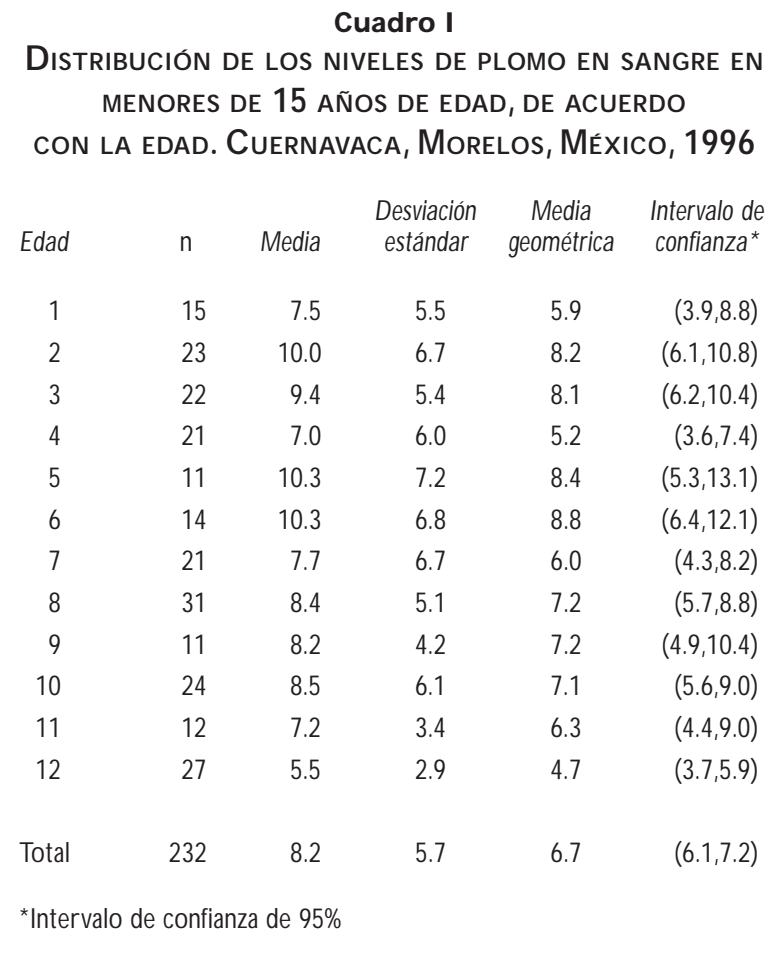

En relación con el tipo de ollas que se utilizan para cocinar los alimentos se encontró una media de logPbS de $2.05 \mu \mathrm{g} / \mathrm{dl}$ en aquellos que utilizan ollas de barro vidriado, en comparación con $1.5 \mu \mathrm{g} / \mathrm{dl}$ en los que utilizan ollas de otro material $(t=-5.59 ; p=<0.05)$. El preparar algunos alimentos en este tipo de material, como guisados, también reportó un valor medio de 2.34 $\mu \mathrm{g} / \mathrm{dl}(t=-4.69 ; p=<0.05)$. El guardar los alimentos en utensilios de barro vidriado, para después comerlos, determinó un valor de $2.22 \mu \mathrm{g} / \mathrm{dl}$ en los niños estudia$\operatorname{dos}(t=-6.30 ; p=<0.05)$. Asimismo, aquellos niños que beben líquidos en este tipo de utensilios reportaron una media $\log \mathrm{PbS}$ de $2.43 \mu \mathrm{g} / \mathrm{dl}(t=-3.8 ; p<0.05)$. La cercanía de un taller mecánico a la casa de los niños fue de $2.19 \mu \mathrm{g} / \mathrm{dl}$ (figura 1, cuadro II).

El modelo final de análisis permitió establecer que los NPS de los niños participantes en este estudio está influido por el nivel de tráfico donde están ubicadas las casas de ellos, dichos niveles son debido a la intensidad del tráfico en las cercanías de la casa del niño $(t=8.35 ; p=0.004)$; el que utilicen barro vidriado para cocinar ( $t=4.51 ; p=0.034)$; la comida se guise en ollas de barro vidriado $(t=8.24 ; p=0.004)$, y que se guarde alimento, para consumo posterior, en recipientes de barro vidriado ( $t=8.36 ; p=0.004)$. La variación de los NPS de este grupo estudiado lo explica el modelo en $28 \%(F=12.44 ; p=<0.05)$.

\section{Conclusiones}

La media de los NPS encontrados en la población aquí reportada resultó menor a los valores reportados por Muñoz (1993), Jiménez (1993) ${ }^{8,9}$ en población pediátrica de la Ciudad de México y son similares a los reportados por Calderón-Salinas (1996) en población pediátrica no expuesta ocupacionalmente, de Coahuila, México. ${ }^{10}$ Aun cuando la media de plomo en sangre reportada sea baja, 29.7\% de los niños rebasaron los 10 $\mu \mathrm{g} / \mathrm{dl}$ lo que, de acuerdo con la Academia Americana de Pediatría, ${ }^{11}$ señala a esta población como socialmente más lábil debido a los efectos que han sido reportados aun en concentraciones más bajas. ${ }^{12}$

En la población pediátrica que estudiamos aún se observan como principales factores que explican los NPS el uso de cerámica de barro vidriado cocido a baja temperatura y la intensidad de tráfico donde viven. La intensidad de tráfico, como una expresión indirecta de emisiones vehiculares de plomo, resultó ser uno de los factores predictores de los NPS en los niños estudiados, pero no con mayor peso que el uso del barro vidriado. Lo anterior podría explicarse en virtud de que en la ciudad de Cuernavaca, hasta 1996, se continuaba distribuyendo gasolina Nova Plus cuyo 
Cuadro II

Distribución de las concentraciones de plomo en SANGRe en menores de 15 años de edad Según diversos factores de riesgo. Cuernavaca, Morelos, México, 1996

\begin{tabular}{|c|c|c|c|c|c|c|}
\hline Variable & $n$ & $\mathrm{PbS}^{*}(\mu \mathrm{g} / \mathrm{dl})$ media & $\mathrm{DE}$ & IC $95 \%$ & Prueba $\mathrm{t}$ & p \\
\hline \multicolumn{7}{|l|}{ Transporte utilizado } \\
\hline Colectivo & 203 & 1.93 & 0.64 & $1.845-2.024$ & -2.2031 & 0.0143 \\
\hline 0 tro & 29 & 1.65 & 0.59 & $1.428-1.883$ & & \\
\hline \multicolumn{7}{|c|}{ Tipo de tráfico donde vive } \\
\hline Intenso & 56 & 2.09 & 0.70 & $1.909-2.286$ & -2.6764 & 0.0080 \\
\hline Bajo & 176 & 1.83 & 0.611 & $1.746-1.928$ & & \\
\hline \multicolumn{7}{|l|}{ Ubicación de la casa } \\
\hline Avenida/carretera & 32 & 2.04 & 0.596 & $1.826-2.256$ & 1.3434 & 0.1805 \\
\hline calle & 200 & 1.87 & 0.649 & $1.786-1.968$ & & \\
\hline \multicolumn{7}{|c|}{ Tiempo de vivir en Cuernavaca ${ }^{\ddagger}$} \\
\hline Cinco y más años & 146 & 1.87 & 0.607 & $1.77-1.97$ & 0.6033 & 0.5469 \\
\hline$<$ de cinco años & 84 & 1.93 & 0.683 & $1.78-2.07$ & & \\
\hline \multicolumn{7}{|c|}{ Tipo de olla para cocinar } \\
\hline Barro vidriado & 155 & 2.05 & 0.619 & $1.958-2.155$ & -5.5973 & 0.0000 \\
\hline 0 tro material & 77 & 1.58 & 0.574 & $1.454-1.715$ & & \\
\hline \multicolumn{7}{|c|}{ Prepara en barro vidriado§ } \\
\hline \multicolumn{7}{|l|}{ Sopa } \\
\hline Sí & 9 & 2.36 & 0.764 & $1.781-2.957$ & -1.5657 & 0.1195 \\
\hline No & 146 & 2.03 & 0.607 & $1.938-2.136$ & & \\
\hline \multicolumn{7}{|l|}{ Guisado } \\
\hline Sí & 58 & 2.34 & 0.62 & $2.17-2.50$ & -4.6975 & 0.0000 \\
\hline No & 97 & 1.88 & 0.55 & $1.77-1.99$ & & \\
\hline
\end{tabular}

Guarda alimentos en utensilios de barro vidriado\#

\begin{tabular}{lrrrrrr} 
Sí & 84 & 2.22 & 0.59 & $2.09-2.35$ & -6.3886 & 0.0000 \\
\hline No & 147 & 1.70 & 0.58 & $1.61-1.80$ & & \\
$\begin{array}{l}\text { Toma líquidos en utensilios de barro vidriado } \\
\text { Sí }\end{array}$ & & & & & & \\
No & 19 & 2.43 & 0.706 & $2.09-2.77$ & -3.8878 & 0.0001 \\
\hline N & 213 & 1.85 & 0.617 & $1.76-1.93$ &
\end{tabular}

Cerca de la casa se encuentra ${ }^{\S}$

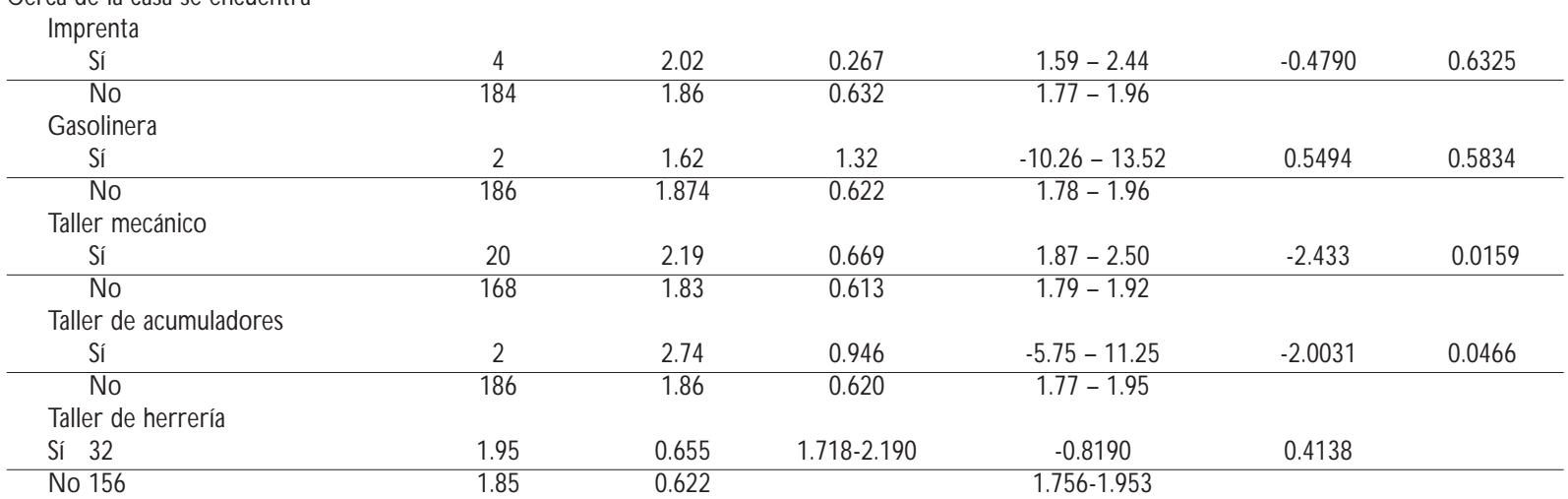

* Logaritmo natural

₹ Sin información: 2 niños

§ Sin información: 77 niños

\# Sin información: 1 niño

\& Sin información: 44 niños

PbS: Plomo en sangre; DE: desviación estándar; IC 95\%: Intervalo de confianza a 905\% 


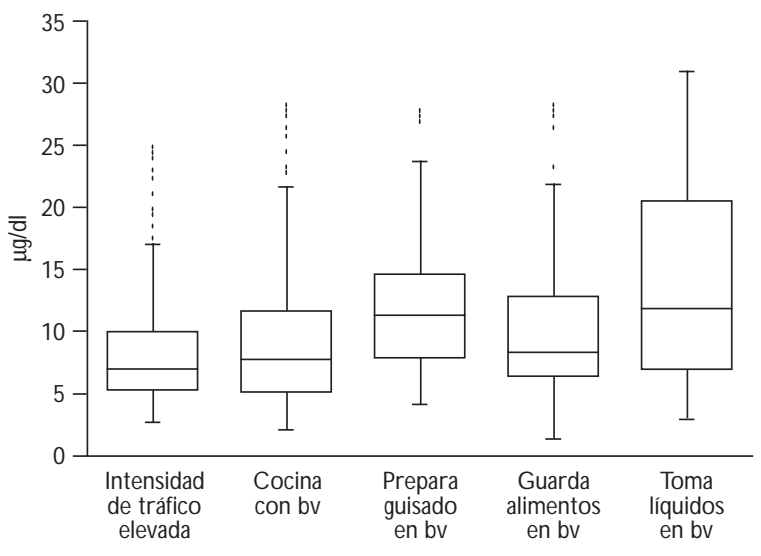

bv $=$ barro vidriado

Figura 1. Distribución de plomo en sangre de ACUERDO CON FACTORES DE EXPOSICIÓN EN 232 NIÑOS menores de 15 años de edAd. Cuernavaca, Morelos, MÉxıCO, 1996

contenido de plomo se estimaba en 0.5 a $1.0 \mathrm{ml} /$ gal de tetraetilo de plomo. ${ }^{13}$ Por el contrario, en otros estudios este factor ya no se reporta como el principal para explicar los NPS de la población, en especial en la Zona Metropolitana de la Ciudad de México, ${ }^{14}$ y otras ciudades como Monterrey y Guadalajara, como lo había sido en fases previas a la reconversión de las gasolinas. ${ }^{15}$ Entre las medidas introducidas para reducir ese tipo de exposición destacan la reducción del contenido de plomo en la gasolina Nova a $0.13 \mathrm{ml}$ de tetraetilo de plomo por galón en 1994, y la introducción de la gasolina Magnasin, cuyo contenido de plomo es de 0.01 g de plomo/gal max. Asimismo la reducción del plomo en otras fuentes donde su contenido era muy elevado (latas, pinturas, etcétera). ${ }^{16}$

Estas acciones han dejado como fuente principal de exposición al barro vidriado con greta base plomo y cocida a baja temperatura, cuyo uso es aún muy extendido por razones económicas y culturales, y, en menor magnitud, otros factores como la exposición paraocupacional, el consumo de tabaco y alcohol. ${ }^{17}$

Morelos es una de las entidades donde, además de esos factores, se realiza la extracción minera de plomo; por lo menos hacia 1993 se registraban un poco más de 700 toneladas de producción de plomo, y en el Valle de Cuernavaca se concentra un buen número de empresas y talleres en donde éste se utiliza. Entre ellas, por lo menos, 29 (9.6\%) producen cerámica y alfarería. Por la información no se logra diferenciar el tipo de greta utilizada y si estos talleres son domésticos o de otro tipo. ${ }^{18}$

Una de las limitaciones del presente trabajo puede ser que los resultados que se reportan tienen un periodo de levantamiento mayor a cinco años y que actualmente la entidad goza del beneficio del cambio de las gasolinas. Hasta el momento no se ha producido algún otro estudio que informe de los NPS en población como la aquí estudiada, por lo que estos resultados podrían servir de referente a futuras investigaciones sobre el tema dentro de la entidad, y en el conjunto de la vigilancia epidemiológica de los NPS en poblaciones pediátricas.

La diversidad de información relativa a exposición al plomo, y los resultados obtenidos por el presente trabajo, indican la necesidad de documentar la presencia de estos factores de exposición a través del inventario de emisiones, monitoreo de niveles de plomo en diversas fuentes, así como en la población, implantación de medidas de intervención directa sobre las fuentes de exposición, y evaluación del impacto.

\section{A gradecimientos}

Agradecemos a la bióloga Verónica Cruz su apoyo en el desarrollo de este proyecto.

\section{Referencias}

1. Conclusiones del Grupo de Trabajo III. CerámicaVidriada. En: Howson $C h$, Hernández-Avila $M$, Rall D, ed. El plomo en A mérica. Estrategias para la prevención. Cuernavaca, Morelos, México: Instituto $\mathrm{N}$ acional de Salud Pública (México),A cademia N acional de Ciencias (USA),

1996:145-149.

2. Juan M, Peña-Corona MP, C astañeda LM. N ormatividad de plomo en México. En: Hernández-Avila M, Palazuelos E, ed. Intoxicación por plomo en México: prevención y control. Perspectivas en salud pública. 21 México 1995:109-120.

3.Torres-Sánchez L, López-C arrillo L, Ríos C. Eliminación del plomo por curado casero. Salud Publica Mex 1999;41supl 2:S106-S108.

4. Grant LD, D avis JM. Effects of low lead exposure on paediatric neurobehavioral development: $C$ urrent findings and future directions. En: Smith MA, Grant LD, Sors AI, Ed. Lead Exposure and child development.An international assessment. D ordrecht, The N etherlands: Klumer Academic Pub 1989:49-115.

5. Secretaría de Hacienda. A genda Estadística 1993. Gobierno del Estado de Morelos, 1994.

6. Intoxicación por plomo: de la detección a la prevención primaria (Indicadores y N oticias de Salud). Salud Publica Mex 1995:37(3) ;264-276.

7. Muñoz I, Romieu I, Palazuelos E, MancillaT, Meneses F, HernándezAvila M. Blood lead level and neurobehavioral development among children living in Mexico City.Arch of Environ Health may/jun 1993;48(3):132-138. 
8. Jiménez C, Romieu I, Palazuelos E, Muñoz I, C ortés M, Rivero A et al. Factores de exposición ambiental y concentraciones de plomo en sangre en niños de la Ciudad de México. Salud Publica Mex 1993;35: 599-606.

9. Jiménez-Gutiérrez C, Romieu I, Ramírez-Sánchez A, PalazuelosRendón E, Muñoz-Q uiles I. Exposición a plomo en niños de 6 a 12 años de edad. Salud Publica Mex 1999; 41 supl 2:S72-S81

10. Calderón-Salinas JV,Valdez-A naya B, Mazuñiga C,Albores-Medina A. Lead exposure a population of Mexican children. Human of Environmental Toxicology 1996; 15: 305-311.

11. N eedleman $\mathrm{HL}$, Bellinger D.The health effects of low level exposure to lead.Ann. Rev Pub Health 1991;18:11-140.

12. Rothenberg SJ, Schnaas L, C ancino-O rtiz S, Perroni-Hernández E, De la Torre $P, N$ eri-Méndez $C$ et al. N eurobehavioral deficits after low level lead exposure in neonates:The Mexico City pilot study. N eurotoxicol Teratol 1989;11:85-93.

13. Heredia-Veloz A, Cibrián-de León P. Evolución de las gasolinas en Pemex a partir de la expropiación petrolera. Rev 0 ctanaje 1996(3).
Disponible en: http://www.franquicia. Pemex. com/octanaje/octxe3.htm. 20.

14. Gobierno del Distrito Federal. Programa para mejorar la calidad del aire en el Valle de México, 1995-2000. México, DF: Gobierno del Distrito Federal, 1996.

15. Romieu I, Palazuelos E, Meneses F, Hernández M.Vehicular traffic as a determinant of blood-lead levels in children:A pilot study in México City. Archives of Environmental Health 1992; 47:246-249.

16. Romieu I, Palazuelos E, H ernández Avila-M, Ríos C, Muñoz Y, Jiménez $C$ et al. Sources of lead exposure in Mexico City. Environ Health Perspect 1994;102:384-389.

17. Junco-Muñoz P, 0 ttman R, Lee J, Barton S, Rivas F, C erda-Flores R. Blood lead concentrations and associated factors in residents of Monterrey, Mexico. Arch Med Res 1996; 27:547.

18. Cámara N acional de la Industria de la Transformación. Directorio Industrial Morelos.1994. México, DF: C anacintra, 1994:102. 